\title{
Data Aggregation in Wireless Sensor Networks
}

\author{
https://doi.org/10.3991/ijoe.v12i11.6233 \\ Luo Li \\ Sichuan Vocational and Technical College of Communication, Sichuan, China
}

\begin{abstract}
In order to solve the problem of low efficiency and high energy consumption in data processing, In this paper, for reducing the communication overhead of data aggregation, we put forward a cluster division based data aggregation scheme for wireless sensor networks in which the cluster is divided into three subareas and some report nodes are assigned to each subarea. Simulation results show that, compared with LEACH algorithm, BP neural network and PSO-BP algorithm, more than 50\% lower compression error is decreased and the simplicity of the improved algorithm makes it suitable for massive and the long term environmental monitoring network.
\end{abstract}

Index Terms - data aggregation, data fusion, trending aggregation algorithm, wireless sensor networks

\section{INTRODUCTION}

As a significant component of Internet of Things, Wireless Sensor Network (WSN) with its advanced information perception, data interaction and other capabilities has attracted extensive attention of the researchers in recent years. WSN consists of a large number of low-power, low-cost, and multi-functional micro sensors which provide data acquisition and information processing service, and also be able to communicate with each other through the wireless channels. Hence, it can form a task-oriented network system on the basis of wireless communication technology and self-organization method. However, densely deployed sensor nodes need to transmit the data information to the user terminal or the control center through the sole sink in the traditional wireless sensor networks. This many-to-one transmission mode put WSNs at enormous risks in many aspects such as energy balancing and reliability. Meanwhile, with the widespread applications of WSN, the single sink node structure cannot meet the demand for rapid development of WSNs[1-2].

With the development of the Internet of Things and wireless sensor network technology, large-scale, highdensity network layout becomes the development trend of wireless sensor networks[3-4]. Comparing to the single sink node wireless sensor network, design of multi-sinks has showed stronger adaptability in the data transmission reliability, node energy balance and other aspects. The packet format conversion contains two steps: (1) SCM analyzes packet according to the format of source packet and gets the payload. (2) SCM packages the payload from steplaccording to the format of goal packet. It will inevitably lead to problems such as address information loss and data rate mismatching to introduce the IEEE 802.11 protocol conversion into the multi-sinks wireless sensor network. To solve the former, this paper introduces a new design of data domain, which divides the data payload into address information and data information. To solve the later, this paper designs a data buffer to queue and temporary store the data packet from different serials. The key design of multi-sinks wireless sensor network based on the protocol is to achieve sink node switching mechanism through the collaborative design of the sink node and sensor node. The mechanism allows the sensor node switch to another sink node to complete data information upload, which its current sink node fails. The design of sensor node and sink node need to match the sink node switching mechanism.

Wireless sensor networks (WSN), attracting plentiful research efforts due to their wide range of potential applications, have been a very active research area. WSN usually consist of a large number of inexpensive sensor nodes that have strictly limited sensing, computation, and communication capabilities. The main tasks for WSN are to collect information from areas under surveillance. It is an important issue to save communication energy, and meanwhile to ensure the sampled data secure. The benefits of in-network processing include minimized amount of data transmission, reduced energy consumption, improved overall bandwidth utilization, and prolonged lifetime. In hostile environments, such as battlefield monitoring and home security, we must take account of the data security, including confidentiality, authentication, integrity, and freshness during transmitting data to the Sink node.

Data aggregation techniques can greatly help conserve the scarce energy resources in sensor networks by minimizing the number of data transmissions. Conventional data aggregation methods are vulnerable, as cluster-heads receive all the data from sensor nodes and then eliminate the redundancy by checking the contents of the data. A secure energy-efficient data aggregation and authentication scheme called SEDAA is presented. Intermediate nodes, i.e. cluster-heads in each level, implement data aggregation based on pattern codes without leaking the contents of the raw data and only distinct data in encrypted form is transmitted from sensor nodes to the base station, so SEDAA is confidential. For reducing the communication overhead of data aggregation, a cluster division based data aggregation scheme for wireless sensor networks in which the cluster is divided into three subareas and some report nodes are assigned to each subarea. The nodes which have same reading and located in same subarea with the report node will keep silent in data aggregating, thus the inner-cluster transmissions is reduced. Analysis and experiments show that the transmissions of innercluster data aggregation of our scheme lower than that of related schemes and the decrease of transmissions is obviously when the redundancy of sensor readings is high.

\section{OVERVIEW}

Data integrity and authentication exploit two main ideas: delayed aggregation and delayed authentication. Instead of aggregation messages at the immediate next hop, messages are forwarded unchanged over the first hop and then 
PAPER

aggregated at the second hop. The $\mu$ TESLA is adopted for authentication of messages transmitted by the base station. Data freshness is gained by using session keys calculated by counters. Moreover, a scalable secure data aggregation and authentication scheme called SSDAA is also presented. The $\mu$ TESLA key chains are used to reveal and authenticate keys locally. At every round, authentication need not wait until aggregation has been completed, so it can be applied to large scale WSN with a little delayed time. Both SEDAA and SSDAA can defend against intruder node attacks and replay attacks, and can limit the effectiveness of compromised node attacks.

Therefore, this paper explains how to solve the above problems by means of increasing the number of sink nodes, so that WSNs becomes more efficient, more stable, more robust, and easier to manage. Zheng's [5] paper focusing on multi-sink node WSNs performs in-depth study from the routing protocol design, multiple sink nodes re-positioning technology and data aggregation strategies respectively, and also proposes relative algorithms. The paper proposes an optimal routing selection algorithm based on fuzzy comprehensive evaluation theory. By fuzzy comprehensive evaluating three factors, hops of sensor node to the sink node, the minimum residual energy of the path, as well as the minimum average link quality, this algorithm provides a distributed optimal multi-path routing corresponding to the sink node which is hierarchical, active and on-demand mixing and QoS in view. In consideration of humane thinking, routing in wireless sensor networks can provide a longer network lifetime, higher packet delivery rate and less routing control information. Hence, in comparison with other multipath routing protocols which don't apply the fuzzy theory and take comprehensive considerations to the effect factors, this algorithm will extend about2.8times of the lifetime of network and increase about14percent of the packet delivery rate on average. But at the same time, it also reduces about 37 percent of the control information during routing process.

Li's [6] paper presents a relocation algorithm based on the centroid principle of multi-sink node. The algorithm utilizes the methods that make one-hop neighbors of the sink node as the system of particles and the number of packets transmitted to the sink node as the particle quality, and regards the multiple sink node as the centroid of each system of particles by means of calculating the centroid position in order to collaboratively approach and pinpoint the their corresponding optimal position. In addition, the optimal position of the sink node can be adjusted according to the changes of network state, thus it can guarantee smaller average number of hops and fewer average node energy. Therefore, compared with the algorithms in which sinks are fixed, the relocation algorithm based on the centroid principle can extend about 69 percent of the network lifetime and improve about 5 percent of the packet delivery rate on average. It also has advantages over other sink relocation algorithms in the network lifetime by about18percent and enhances about6percent of the success rate of data transmission on average.

Niu's [7] paper proposes a data aggregation algorithm which is able to balance the average energy consumption of sensor nodes and average data transmission delay. It first makes use of secondary fuzzy comprehensive evaluation to make relevant adjustment about the number of packets forward in a period through the original optimal routing to fit for data aggregation. As a result, new data forwarding paths can more efficiently balance energy consumption of the sensor node and improve data transmission reliability, as well as improving data aggregation performance by increasing the overlap of the original paths. Moreover, the algorithm also can utilize the states of the sensor nodes to dynamically adjust its current wait time of aggregation, thus the data aggregation timing mechanism can effectively balance the average energy consumption of each sensor node, and reduce average data delay in network. Therefore, compared with algorithms that not involve data aggregation, it can extend about one times of the network lifetime, but will reduce about 3 percent of the network packet delivery rate on average. It can also achieve better lifetime than other data aggregation algorithms by about 10 percent, and improve about 6 percent of the packet delivery rate and about 16 percent of the aggregation rate as well as reducing about6percent of the average transmission delay.

Based on the above achievements, Huang [8] designs and implements a data compression based monitor prototype system. The system architecture consists of three layers: sensor networks tier, data service tier, application tier. On the basis of Tiny OS, the sensor networks tier implements a data sampling module, a clustering and routing protocol, and some data compression algorithms in nesC. The sensor networks tier runs in Micaz mote. All compressed sensing data are transmitted to the gateway mote. The gateway mote is connected to the on-site PC via USB port. The data service tier is a middleware for message/data exchange between application tier programs and the WSN gateway mote. The application tier is implemented on the on-site PC or remote client PC. Its functions include local or remote monitoring, data analysis and visualization.

By designing an incremental algorithm for computing regression coefficients, a self-adaptive regression-based multiple-streams wavelet compression algorithm with infinite norm error bound (AR-MWCEB) is proposed in Yang's paper [9]. Based on error bounds and compression gains, the self-addictiveness means that our algorithms make decisions automatically to transmit raw data or regression coefficients and to select the number of data involved in regression. Theoretically and experimentally, it is concluded that the proposed algorithms can effectively exploit the temporal and multiple-dimension-stream correlations on the single sensor node and exploit the temporal and spatial correlations among multiple streams on the cluster head and achieve a significant data reduction. Furthermore, we observe that the algorithms are also pretty good when multiple-streams correlations are reduced or non-stationary.

\section{Method AND ALGORITHM}

Conventionally, multipath is viewed as an unwanted feature of wireless communications hence; some means are used to mitigate its bad-effects such as equalization, and diversity arrays. The utilization of relays has been considered to offer capacity improvement and coverage extension in wireless schemes. By transmitting the signal throughout multiple relay hop channels, it could be possible to decrease the path loss and hence to accomplish higher throughput. Contrary to what happen in single-hop transmission, it is attractive to balance the capacity between mufti-hop channels in relay communication, since 
the end-to-end capacity is subject to issue of the minimum capacity of hop channel. The imbalance capacity between multi-hop could be mitigated by means of power allocation. It is of major concern how to adjust the transmit power of each relay terminal due to the change of charnel condition. The utilization of multiple at both transmitter and receiver has really enhanced the spectral efficiency and dependability of wireless communication schemes. The so-called MIMO system uses spatial resources as well as resources in frequency and time domain.

Through allocation of the transmit power on the basis of the average channel gain; then the total amount of transmit power can be reduced for given end-to-end bit error rate in addition to symbol error rate (SER). Also, the transmit power could be allocated on the basis of the Signal-to-Noise (SNR) to achieve the maximum end-to-end capacity in the dual-hop single-input single-output (SISO) relay channels. Communication is assumed to be conducted in long bursts having a lot of symbols in order that the time horizon information, which concerning theoretic description of communications will address a meaningful idealization.

Nevertheless, the bursts are supposed to be short adequate that the rapid channel is basically unchanged during a burst. In a high data rate scheme, MIMO antennas are utilized; the bit rate might run to a few Mbps, while the fading rate will be within a few hundred Hz. Thus, the basic vector equation that related to the input and output complex envelopes will be written as

$$
X^{(1)}(k)=\sum_{i=1}^{k} x^{(0)}(i)
$$

Variable $x^{(0)}$ has the original data series $x^{(0)}=\left[x^{(0)}(1), x^{(0)}(2), \ldots, x^{(0)}(n)\right]$, with a 1 -AGO order to generate an accumulated generating sequence $x^{(1)}=\left[x^{(1)}(1), x^{(1)}(2), \ldots, x^{(1)}(n)\right]$.

It means that charm quality attributes over time will gradually become the desired quality, and finally becoming a basic quality.

$$
\frac{d x^{(1)}}{d t}+\alpha x^{(1)}=\mu
$$

In the formula, $\alpha$ and $\mu$ are parameters. $\mu$ is the control. Solution of the differential equation as follows:

$$
x^{(1)}(k+1)=\left[x^{(0)}(1)-\frac{\bar{\mu}}{\bar{\alpha}}\right] e^{-\bar{\alpha} k}+\frac{\bar{\mu}}{\bar{\alpha}}
$$

Among them, $\bar{\alpha}$ and $\mu$ is the approximate solution of formula (2), according to the least squares method to obtain:

$$
\begin{aligned}
{\left[\begin{array}{l}
\bar{\alpha} \\
\bar{\mu}
\end{array}\right] } & =\left(B^{T} B\right)^{-1} B^{T} Y \\
Y_{n} & =\left[\begin{array}{l}
x^{(0)}(2) \\
x^{(0)}(3) \\
\cdots \\
x^{(0)}(4)
\end{array}\right]_{n-1}
\end{aligned}
$$

Formula (5) is called GM $(1,1)$ model of response time function model, it is a specific formula for calculat- ing GM $(1,1)$ model of gray prediction, this type do regressive reduction, and gray had predicted the number of columns in the original model:

$$
\bar{x}^{(0)}(k+1)=\bar{x}^{(1)}(k+1)-\bar{x}^{(1)}(k)(6)
$$

PSO particle optimization algorithm basic formula is as follows:

$$
\begin{array}{r}
v_{i}^{n+1}=w v_{i}^{n}+c_{1 i} \times r_{1} \times\left(P_{i}^{n}-X_{i}^{n}\right)+c_{2 i} \times r_{2}\left(G^{n}-X_{i}^{n}\right) \\
(7) \\
X_{i}^{n+1}=X_{i}^{n}+v_{i}^{n}(8)
\end{array}
$$

so as to minimize the mean square error indicators:

$$
E(X)=\frac{1}{2 N} \sum_{p=1}^{n} \sum_{k=0}^{c}\left(Y_{k, p}(X)-t_{k, p}\right)
$$

Under the linear relationship, basic equation is shown in equation (2):

$$
\partial_{j}\left(e_{i j k l} \partial_{k} u_{l}-\eta_{k i j} \partial_{k} \varphi\right)=0 \text { (10) }
$$

The linear differential equation can be expressed into the following simplified forms:

$$
\begin{aligned}
& L(\nabla, \omega) f(x, \omega)=0, \\
& \quad L(\nabla, \omega)=T(\nabla)+\omega^{2} \rho \mathrm{J}
\end{aligned}
$$

In which,

$$
\begin{gathered}
T(\nabla)=\left\|\begin{array}{cc}
T_{i k}(\nabla) & t_{i}(\nabla) \\
t_{k}^{T}(\nabla) & -\tau(\nabla)
\end{array}\right\|, \mathrm{J}=\left\|\begin{array}{cc}
\delta_{i k} & 0 \\
0 & 0
\end{array}\right\|, \\
f(x, \omega)=\left\|\begin{array}{c}
u_{k}(x, \omega) \\
\varphi(x, \omega)
\end{array}\right\| \\
T_{i k}(\nabla)=\partial_{j} C_{i j k l} \partial_{l}, \quad t_{i}(\nabla)=\partial_{j} e_{i j k} \partial_{k}, \\
\tau(\nabla)=\partial_{i} \eta_{i k} \partial_{k}
\end{gathered}
$$

Consider an infinite situation, we have the equation (5) in the following:

$$
L^{0}=\left\|\begin{array}{cc}
C_{i j k l}^{0} & e_{k i j}^{0} \\
e_{i k l}^{0 T} & -\eta_{i k}^{0}
\end{array}\right\|
$$

Consider the propagation, instead the equation (13) with the following form:

$$
\begin{gathered}
C(\mathrm{x})=C^{0}+C^{1}(\mathrm{x}), \quad e(\mathrm{x})=e^{0}+e^{1}(\mathrm{x}) \\
\eta(\mathrm{x})=\eta^{0}+\eta^{1}(\mathrm{x}), \quad \rho(\mathrm{x})=\rho_{0}+\rho_{1}(\mathrm{x})
\end{gathered}
$$

Then we have equation (15) to (18):

$$
\begin{aligned}
& C^{1}=C-C^{0}, \quad e^{1}=e-e^{0}, \\
& \quad \eta^{1}=\eta-\eta^{0}, \quad \rho_{1}=\rho-\rho_{0}
\end{aligned}
$$

The containing inclusions can be simplified into the following integral equation set:

$$
\begin{aligned}
& f(x, \omega)=f^{0}(x, \omega)+\int_{V} S\left(x-x^{\prime}\right)\left(\mathrm{L}^{1} F\left(\mathrm{y}^{\prime}\right)\right. \\
& \left.+\rho_{1} \omega^{2} \mathbf{g}(R) \mathrm{T}_{1} f\left(\mathrm{y}^{\prime}\right)\right] S\left(\mathrm{y}^{\prime}\right) \mathrm{dy}^{\prime}
\end{aligned}
$$

Therefore, any working can only accomplish a bit rate, at a number of desired small bit error rates, which is only a portion of the information theoretic capacity. As a result of the random phenomenon of the matrix transfer function 
$\mathrm{H}$, hence the capacity is as well is a random quantity. However, that $\mathrm{H}$ sends an $\mathrm{M} \times \mathrm{N}$ matrix in which $\mathrm{M}$ is the number of receiving elements along with $\mathrm{N}$ is the number of transmitting elements. The expression in equation (11) proposes that the capacity of an $(\mathrm{N}, \mathrm{M})$ scheme is equivalent to the capacity of an $(\mathrm{M}, \mathrm{N})$ scheme.

Network management problems, such as routing and resource allocations in a wireless ad-hoc network are very much more challenging when compared to other's wireless networks like cellular networks. Hence, by clustering the terminals in the complete network, a better radio resource usage can be achieved. Not only routing based on clustering decreases the quantity of information transmitted in the network but also the routing delays. Clustering mobile ad-hoc network is very important, because it can be utilized to control. The spatial reuse of the shared channel, so as to reduce the quantity of data which will be exchanged with a view to maintain routing as well as control information within a mobile surroundings, besides, building and maintaining cluster on the basis of virtual network architectures. Therefore, in this section a wireless clustering ad-hoc network is proposed to reduce the huge amount of information that has to be sent from the disaster situation to the disaster authorities, such as rescue team, police men, and fire department. In the proposed network, the terminals are divided into groups known as clusters.

\section{EXPERIMENT RESULT}

We should model the routing of data aggregation in wireless sensor networks as a tree, such as VPCS (Virtual Polar Coordinate System). The sensor nodes deployed in the wide area, will sensed gathered data to the sink node. All the paths from node to sink node comprised a tree routing, such as Figure 1.

If the node cannot communicate to the node of near zone, it can find the node of the same zone as the father node, such as Figure 2.

Assume $\mathrm{E}$ as the threshold of the compression algorithm, set $E \min <E<E m a x$, and set Eadj as the adjustment, set $\mathrm{T}$ as the tolerance of the rate of change of compression interval. Define MTC as the maximum time span of the compression interval to store data in case the monitoring data changes slowly, and adjust T and MTC accordingly. The specific steps are as follows:

Step 1: initiate parameters at the beginning of the algorithm. Set $\mathrm{E}$ as the average of the upper and lower threshold, $E=($ Emin + Emax $) / 2$. Set Eadj and $T$ as $($ Emin+Emax $) / 20,0.2$ respectively.

Step 2: start gathering data and computing the interval time of the compression. If the time between the start point and the data point is greater or equal to MTC, then end the compression compulsively, and move to step 4 .

Otherwise move to step 3.

Step 3: start compressing by calculating the slope of the upper and lower doors. Compare the slope with the maximum of the previous upper door and the minimum of the previous lower door, and take the maximum slope of the two upper doors and the minimum of lower doors. If the slope of the upper door is greater than the lower door, then store the previous time mark and calculate the slope of the fitting line $k=(k 1+k 2) / 2$. End the current compression interval and move to step 4. Otherwise add 1 to the length of the current compression interval and move to step 2 .

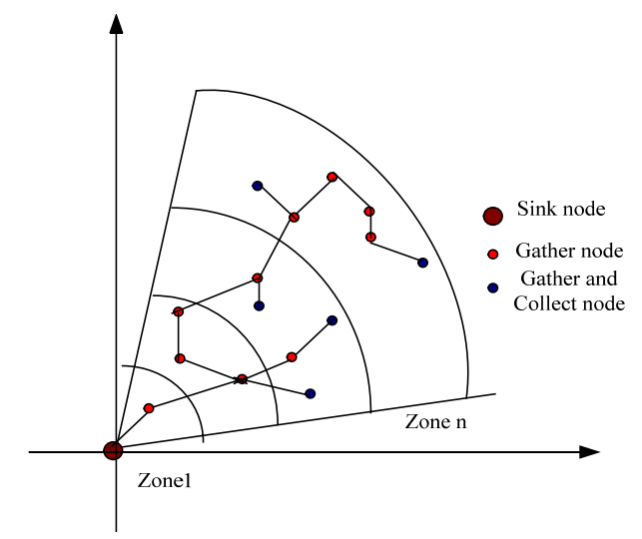

Figure 1. The data aggregation of wireless sensor networks

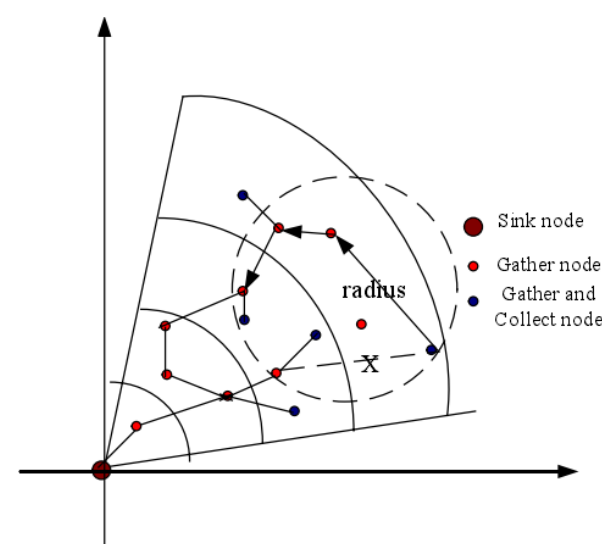

Figure 2. Choose the father node in the same zone by radius

Step 4: at the end of the compression, calculate the length of the current interval and the rate of the change of the length a and make the following judgment:

a) If- $\mathrm{T}<\mathrm{a}<\mathrm{T}$, then the variation of the current data is within tolerance and make no adjustment to $\mathrm{E}$.

b) Otherwise, then the variation of the current data flattens and $\mathrm{E}$ is too large, which may lead to larger error of the data compression. Adjust E within Emin.

Figure 3 shows the scene at node 100, the number of nodes alive COA-BP and LEACH, BP, GA-BP algorithm comparing changes over time. Figure 4 shows the Sink node received under BP algorithm and LEACH algorithm, GA-BP algorithm and COA-BP algorithm. The energy consumption of those four algorithms was compared based on the analyzing of each energy cost. The remaining average energy of four algorithms node are shown in Figure 5 .

Assuming simulation environment randomly throw 100 sensor nodes in the $100 \mathrm{~m}^{*} 100 \mathrm{~m}$ observation area. The simulation specific parameters are set as follows: node initial energy $1 \mathrm{~J}$, random deployment of network topology, node communication range of SOM, the energy consumption for the transmission of data $45 \mathrm{~nJ} / \mathrm{bit}$, receive data consumption is $30 \mathrm{~nJ} / \mathrm{bit}$, packet size 64 bytes, channel fading parameter of 0.3 , the simulation time is $300 \mathrm{~s}$. BP algorithm for the maximum number of iterations Epochs is 2000. Experimental simulation computer is configured Pentium processor, with Matlab R2012b programming. In this paper, the life cycle and the number of packets in four protocol algorithm for wireless sensor networks LEACH, BP, GA-BP, COA-BP algorithm received Sink node, the overall energy consumption compared. 
PAPER

DATA AgGREgAtion IN WIRELESS SENSOR NETWORKS

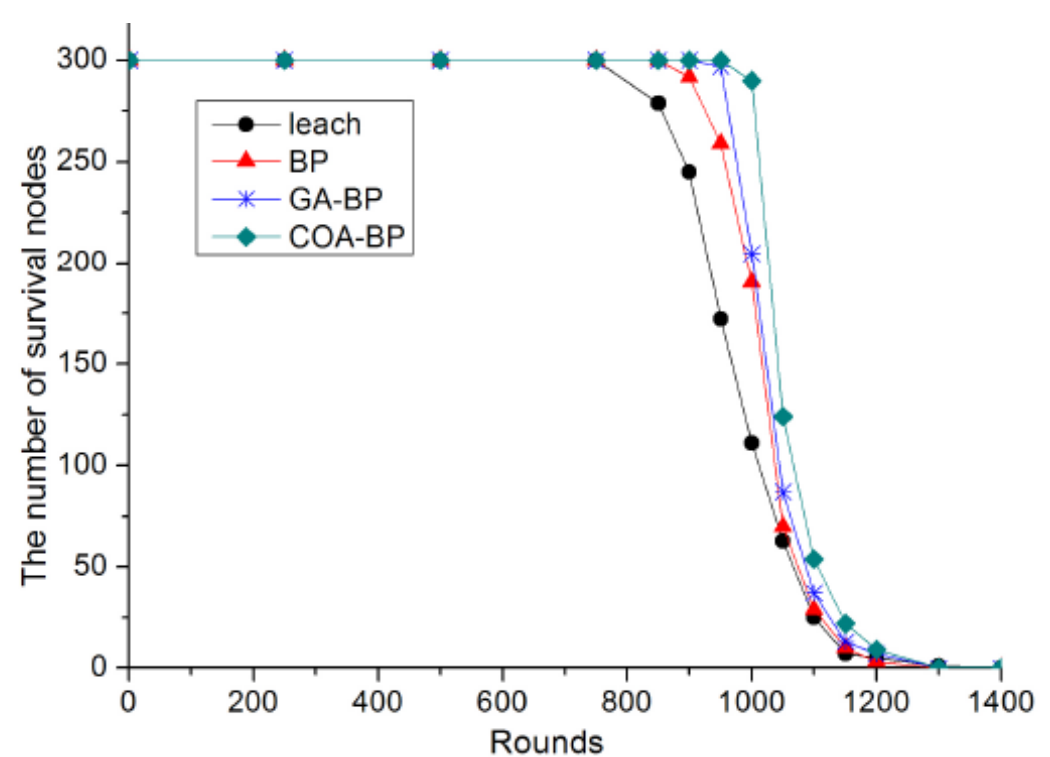

Figure 3. The COA-BP and LEACH, BP, GA-BP algorithm comparing

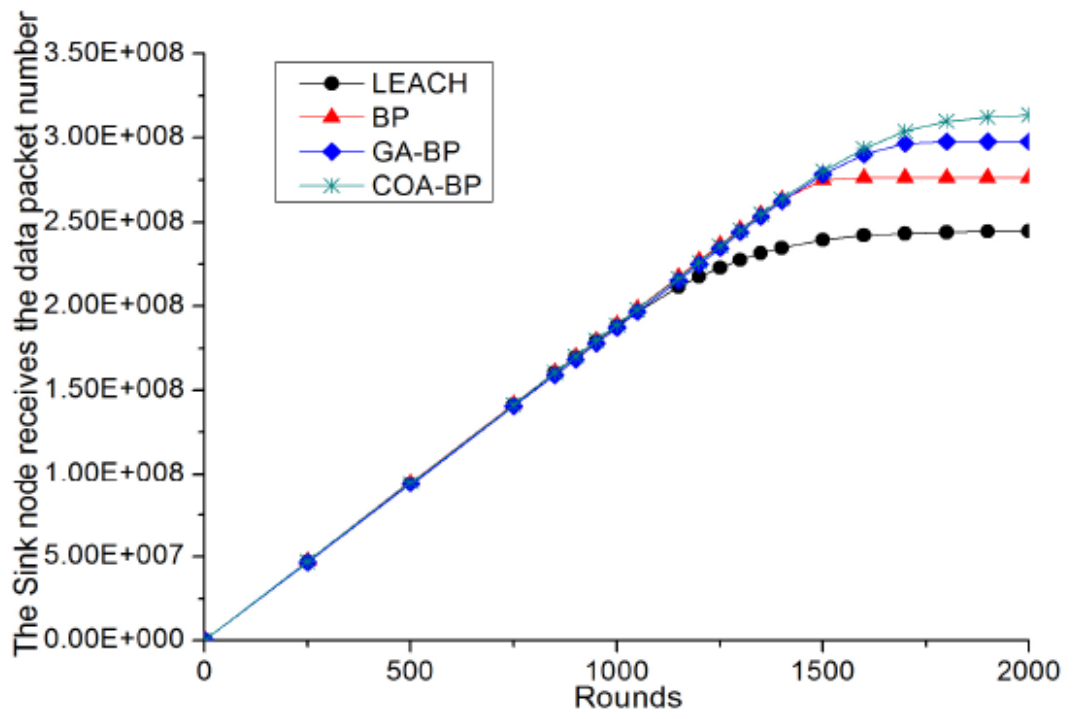

Figure 4. The Sink node received under BP algorithm and LEACH algorithm

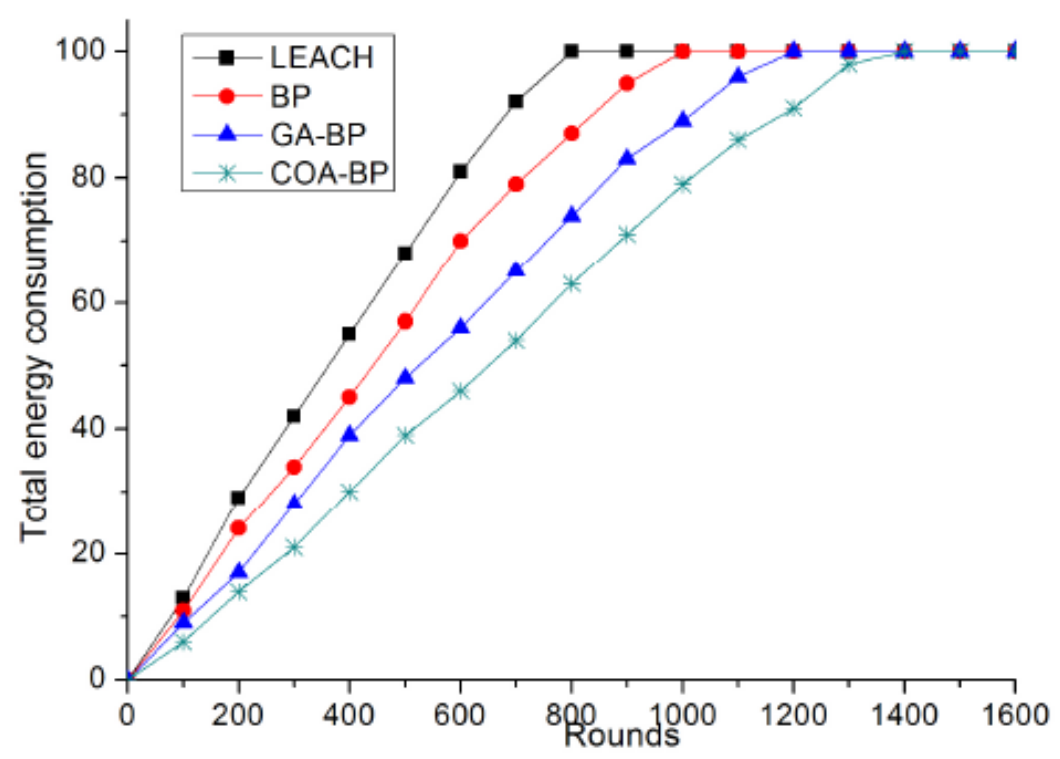

Figure 5. The remaining average energy of four algorithms node 


\section{DISCUSSION}

As can be seen from the figure, before the number of poll are 800 , the number of their survival nodes are basic similar, are not how they change, but the number is 800 1100 polling period is evident, LEACH algorithm biggest energy-consuming, was straight decline, BP algorithm is relatively more stable, GA-BP relative to the previous two smaller energy consumption. The COA-BP algorithm which is proposed in this paper is the lowest power consumption, the number of life-cycle is the longest, and the relative fusion accuracy is the best.

Through the comparison of packets received by the Sink of the four algorithms, it is concluded that COA-BP algorithm can reduce the amount of data in the network through the effective data fusion, and it is also proved that COA-BP algorithm has a longer survival time. Based on the energy-efficient in data transmission, as time increases, the packet COA-BP received is much more than the packet LEACH,BP and GA-BP algorithm protocol received. The packet traffic is also more stable.

\section{CONCLUSION}

In order to solve the problem of low efficiency and high energy consumption in data processing, in this paper, for reducing the communication overhead of data aggregation, we put forward a cluster division based data aggregation scheme for wireless sensor networks in which the cluster is divided into three subareas and some report nodes are assigned to each subarea. In order to solve the problem of low efficiency and high energy consumption in data processing, In this paper, for reducing the communication overhead of data aggregation, we put forward a cluster division based data aggregation scheme for wireless sensor networks in which the cluster is divided into three subareas and some report nodes are assigned to each subarea. The main tasks for WSN are to collect information from areas under surveillance. It is an important issue to save communication energy, and meanwhile to ensure the sampled data secure. The benefits of in-network processing include minimized amount of data transmission, reduced energy consumption, improved overall bandwidth utilization, and prolonged lifetime. In hostile environments, such as battlefield monitoring and home security, we must take account of the data security, including confidentiality, authentication, integrity, and freshness during transmitting data to the Sink node. Data aggregation techniques can greatly help conserve the scarce energy resources in sensor networks by minimizing the number of data transmissions. Conventional data aggregation methods are vulnerable, as cluster-heads receive all the data from sensor nodes and then eliminate the redundancy by checking the contents of the data. Simulation results show that, compared with LEACH algorithm, BP neural net- work and PSO-BP algorithm, more than 50\% lower compression error is decreased and the simplicity of the improved algorithm makes it suitable for massive and the long term environmental monitoring network.

\section{REFERENCES}

[1] J. Yang, R. Xu, Z. Lv, et al., "Analysis of Camera Arrays Applicable to the Internet of Things," Sensors, vol. 16, no.3, March 2016. https://doi.org/10.3390/s16030421

[2] Y. Hu and X. Zhang, "Aggregation Tree Based Data Aggregation Algorithm in Wireless Sensor Networks," International Journal of Online Engineering, vol. 12, no 06, pp. 10-15, June 2016. https://doi.org/10.3991/ijoe.v12i06.5408

[3] H. Jing, "Node deployment algorithm based on perception model of wireless sensor network," International Journal of Automation Technology,vol.9, no.3, pp. 210-215, April 2015. https://doi.org/10.20965/ijat.2015.p0210

[4] H. Jing, "Routing optimization algorithm based on nodes density and energy consumption of wireless sensor network," Journal of Computational Information Systems, vol. 11, no.14, pp. 50475054, July 2015.

[5] J. Zheng, et al., "Auction-based adaptive sensor activation algorithm for target tracking in wireless sensor networks," Future Generation Computer Systems, vol. 39, no. 1, pp.88-99, 2014. https://doi.org/10.1016/j.future.2013.12.014

[6] Z. Li, et al., "A low latency, energy efficient MAC protocol for wireless sensor networks," International Journal of Distributed Sensor Networks, vol. 10, no. 6, pp.1-9, 2015. https://doi.org/10.1155/2015/946587

[7] J. Niu, et al., "R3E: Reliable Reactive Routing Enhancement for Wireless Sensor Networks," IEEE Transactions on Industrial Informatics, vol. 10, no. 1, pp.784-794, 2014. https://doi.org/10.1109/TII.2013.2261082

[8] C. Huang, Y. Tseng, and L. Lo, "The coverage problem in threedimensional wireless sensor networks," Journal of Interconnection Networks, vol. 08, no. 03, pp. 3182-3186, 2015.

[9] H. Yang, et al., "Toward resilient security in wireless sensor networks," Proceedings of the 6th ACM international symposium on Mobile ad hoc networking and computing ACM, pp.34-45, 2015.

[10] Y. Li, Y. Zhang, J. Chen, et al., "Improved Compact Polarimetric SAR Quad-Pol Reconstruction Algorithm for Oil Spill Detection," IEEE Geoscience \& Remote Sensing Letters, vol. 7, no.1, pp. 1139-1142, 2014. https://doi.org/10.1109/LGRS.2013.2288336

[11] B. B. Reddy, and K. K. Rao, "A Modified clustering for LEACH algorithm in WSN," International Journal of Advanced Computer Science \& Applications, vol. 4, no.5, 2013.

\section{AUTHOR}

Luo Li is withSichuan Vocational and Technical College of Communication, Sichuan, China (e-mail: 48697698@qq.com).

The paper is supported by the National Natural Science Foundation of China (NO. 61573081) and The Technology Research about Network Equipment Cooperative and Active Service on Smart Home base on Internet of Things (NO. 2012RZY01). Submitted 09 September 2016. Published as resubmitted by the author 10 October 2016. 